\title{
The retinopial vein: a vein passing directly from the retina to the pia mater at the optic nerve head
}

\author{
Gordon Ruskell
}

\begin{abstract}
Backgroundlaims-All blood drainage from the retina is thought to occur through the central retinal vein with rare, if any, exceptions. A chance observation of a specimen in which this pattern did not apply suggested that the subject required review.

Methods-An optic nerve head, identified from an earlier study as possessing an unconventional venous drainage arrangement, was examined histologically using interrupted serial resin sections. 200 fundus photographs were examined to compare with the results from the sectioned optic nerve head.

Results-A retinopial vein passing from the temporal retina and entering the pia mater without first joining the central retinal vein was observed. Two of the fundus photographs and possibly four others displayed a venous pattern consistent with this arrangement.

Conclusions-The retinopial vein complements the central retinal vein in blood drainage from the retina. Uncertainty in identifying the vein ophthalmoscopically prevents a reliable estimate of its incidence but it appears to be uncommon. Retinal dysfunction may be limited in cases of central retinal vein occlusion in the presence of a retinopial vein. (Br f Ophthalmol 1998;82:495-497)
\end{abstract}

Retinal veins converge on the optic nerve head forming the central retinal vein which is exclusively responsible for conducting blood from the retina. This statement expresses current understanding of retinal venous vasculature apart from rare instances of a cilioretinal vein when presumably a fraction of blood drains to the choroid. ${ }^{1}$ In a recent histological survey of the optic nerve head a different arrangement was observed in one of the specimens used. ${ }^{2} \mathrm{~A}$ substantial vein passed directly to the pia mater without first joining the central retinal vein. The vein, for which the name retinopial is appropriate, was thought worthy of a short report.

Applied Vision

Research Centre, Department of Optometry and Visual Science, City University, 311-321 Goswell Road, London EC1 7DD G Ruskell

Accepted for publication 9 December 1997 removed with a collar of sclera, choroid, and retina attached. The nerve was washed overnight, immersed in an unbuffered solution of $1 \%$ osmium tetroxide for 1 hour, washed briefly, dehydrated in graded ethanols, cleared in xylene, and embedded in Araldite.

Longitudinally cut serial sections $1 \mu \mathrm{m}$ thick were prepared using an ultramicrotome and every fifth section retained. Resin was removed from the sections with sodium methoxide and stained in $1 \%$ toluidine blue in an equal volume of sodium carbonate and examined with a light microscope.

Two hundred fundus photographs obtained from clinical records were inspected to seek correlates of the histological appearance of the optic nerve head. They were the most recently obtained photographs of patients without eye pathology; otherwise they were unselected.

\section{Results}

Retinal veins converged on the optic nerve head, forming two vessels at the level of the lamina cribrosa, becoming a single central retinal vein about $2 \mathrm{~mm}$ from the disc surface. A further vein turned sharply from the temporal retina $0.15 \mathrm{~mm}$ from the disc margin, entered the lamina cribrosa, and then turned towards the nerve periphery. After a short undulating course it entered the pia mater $0.4 \mathrm{~mm}$ from the cul de sac at the termination of the leptomeninges (Fig 1). At the retina, the maximum width was $68 \mu \mathrm{m}$ and the vessel showed little change in width in its course through the nerve apart from slight reductions at each turn, until widening slightly to a maximum of $84 \mu \mathrm{m}$ at the pia mater where it received two small pial veins. For comparison, the maximum width of the central retinal vein measured $108 \mu \mathrm{m}$ close to its formation central to the lamina cribrosa.

In two fundus photographs, a vein crossed from the temporal retina to the disc and disappeared abruptly as it turned sharply to become buried in the deep tissues of the nerve head remote from the main disc veins and close to the disc margin; one of them is shown in Figure 2. These are the predictable ophthalmoscopic characteristics of the retinopial vein. Four other photographs displayed a vein disappearing from view in the same manner but closer to the main veins and whether or not they might have joined them was equivocal.

Shortly after being alerted to the histological evidence of the retinopial vein, Professor Berndt Ehinger photographed the fundi of a patient with no known retinal pathology, each possessing a putative retinopial vein. One of the photographs is reproduced in Figure 3 with his permission. The vein of the other retina occupied a similar position. 

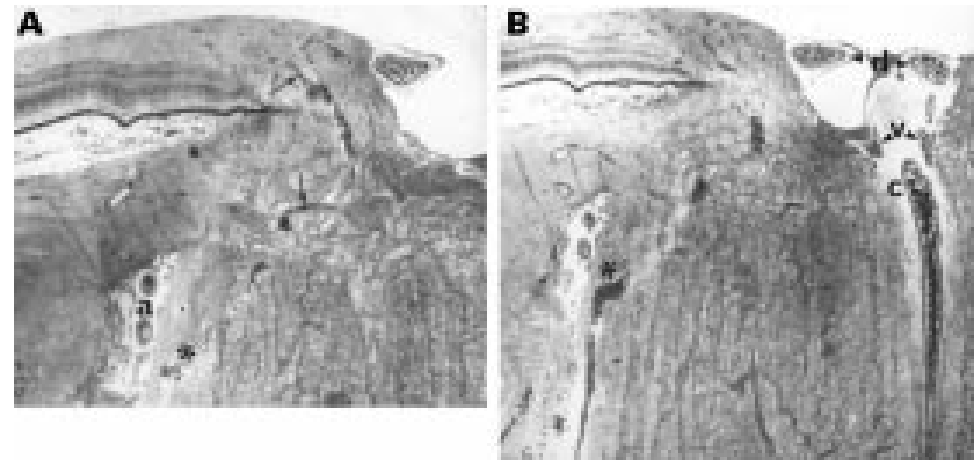

Figure 1 Two micrographs to show most of the course of a vein passing from the retina (arrows) through the lamina cribrosa to the pia mater (asterisks). a= subarachnoid space, $c=$ central retinal artery, $d=$ divisions of the central retinal artery, $v=$ divisions of the central retinal vein. The sections were separated by approximately $25 \mu \mathrm{m}$.

\section{Discussion}

There can be little doubt that the vessel of the histological study drained blood from the retina directly to the pia mater. Material was not available to trace the further course of the retinopial vein. Its size, although small compared with the central retinal vein, was large enough to have drained a substantial area of the temporal retina. There is some doubt as to whether the retina was free of pathology, even though none was reported, as deep cupping of a small area of the disc was present. However, the possibility that the retinopial vein represented a pathological development is unlikely. Where development of supplementary venous drainage from the eye at the disc has been reported, drainage is to the choroid via optociliary veins, not to the optic nerve, and is regarded as a response to obstruction of venous blood flow from the retina..$^{3-5}$ Moreover, it is associated with a lengthy history of blindness.

A recently reported superior hemiretinal retinal vein that appeared ophthalmoscopically to enter the choroid adjacent to the $\operatorname{disc}^{6}$ has some similarities to the retinopial vein. Retroocular Doppler images attributable to the vein were detected in the perineural area at the level of the lamina cribrosa, continuing down the side of the optic nerve, presumably in the pia mater. This vessel might be regarded as a variant of the retinopial vein, differing only in its position of exit from the retina.

A ready explanation for the existence of retinopial veins cannot be found from a consideration of the development of the retinal vasculature. The central retinal vein often has two trunks at the disc that unite while within the optic nerve at a variable distance from the disc as a congenital anomaly, ${ }^{78}$ the vein being formed by the coalescence of two separate vessels in early intrauterine life. ${ }^{9}$ Although there is no evidence that separate trunks leave the eye other than at the centre of the disc, it is feasible that one of the original trunks may become displaced and persist. This is an unlikely explanation for the histological example presented because there were two venous trunks at the disc in this specimen, uniting $2 \mathrm{~mm}$ behind the disc.

Assuming that the retinopial vein can occur as a normal variant, it would be of value to know its incidence and with what confidence

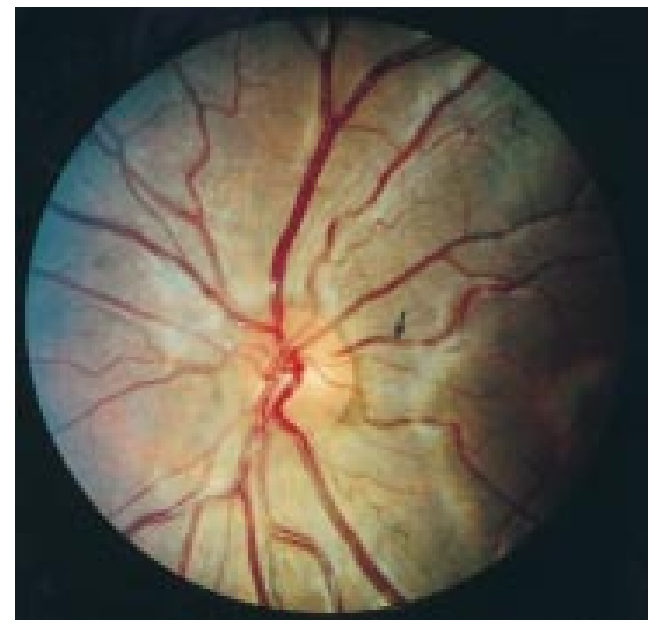

Figure 2 Fundus photograph of a left eye with a temporal vein passing from the macula region and becoming lost to view shortly after crossing the disc margin nearly half a disc diameter from the major vessels.

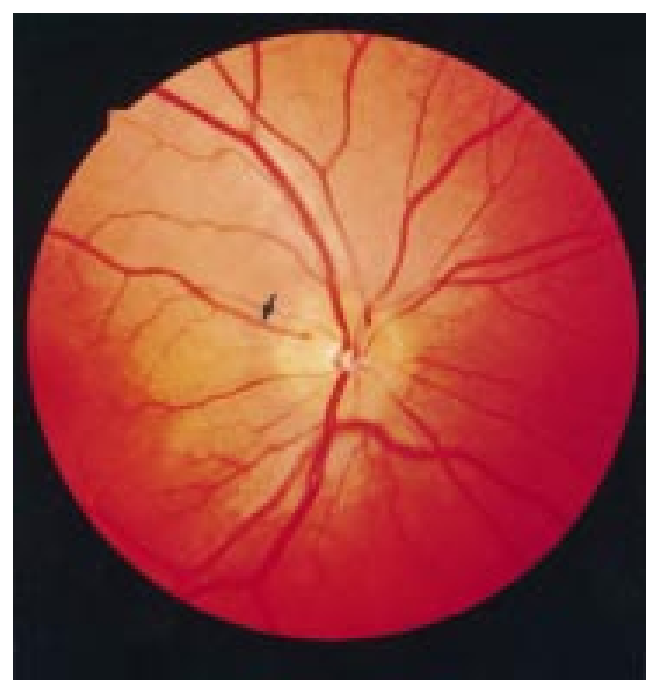

Figure 3 Fundus photograph of a right eye showing a putative retinopial vein in a similar position to that of Figure 2.

one might recognise the vessel ophthalmoscopically. The fundus photograph survey provides tentative answers to these questions. The two photographs displaying vessels fully consistent with the criteria proposed for retinopial veins represent only $1 \%$ of those examined, and another $2 \%$ offered more tenuous evidence for presence of the vessel. In the remainder, all disc veins joined or appeared to be about to join the central retinal vein.

Hitherto, veins meeting the ophthalmoscopic criteria described here, would naturally be considered to join the central retinal vein, or be confused with cilioretinal veins. These rare vessels drain blood from the retina to the choroid and were observed only twice in a survey of 1000 eyes. ${ }^{10}$ However, the retinopial vein does not hook around the disc margin in the manner of the cilioretinal vein and is lost to view some distance from the margin, suggesting that drainage is not to the choroid. Clearly, ophthalmoscopy alone is an inadequate determinant of the drainage path taken when veins appear to leave the disc well removed from its 
centre but, notwithstanding, the present study indicates that in assessing the vasculature of the optic disc, one should consider the possibility of the presence of a retinopial vein.

Visual loss due to occlusion of the central retinal artery, with retention of visual field relating to retinal areas served by the more common cilioretinal artery is well documented $^{11112}$ and hemicentral retinal vein occlusion, where separate venous trunks are present at the disc is reported. ${ }^{7}$ Similarly, limitation of retinal dysfunction resulting from occlusion of the central retinal vein is feasible in the presence of a retinopial vein, whether the occlusion lies at the disc or further back in the optic nerve. Equally, selective occlusion of the retinopial vein could account for a proportion of those frequent cases where only the macula area is affected. ${ }^{13}$

I am grateful to Professor W Lee of Glasgow and Professor B Ehinger of Lund for the provision of material.

1 Wise G, Dollery C, Henkind P. The retinal circulation. New York: Harper \& Row, 1971:298.
2 Ruskell GL. Peripapillary venous drainage from the choroid: a variable feature in human eyes. Brf Ophthalmol 1997; 81: 76-9.

3 Frisén L, Hoyt WF, Tengroth BM. Optociliary veins, disc pallor and visual loss: a triad of signs indicating spheno-orbital meningioma. Acta Ophthalmol 1973;51: 241-9.

4 Rodrigues MM, Savino PJ, Schatz NJ. Spheno-orbital meningioma with optociliary veins. Am $\mathcal{F}$ Ophthalmol 1976;81: 666-70

5 Zakka KA, Summerer RW, Yee RD, et al. Optociliary veins in a primary optic nerve sheath meningioma. Am $f$ Ophthalmol 1979;87:91-5.

6 Williamson, TH, Barr, DB, Baxter, GM. Understanding of the retinal circulation provided by an anomalous vein. $B r \mathcal{F}$ Ophthalmol 1994,78:798-9.

7 Hayreh SS, Hayreh MS. Hemi-central retinal vein occlusion. Arch Ophthalmol 1980;98:1600-9.

8 Chopdar AC. Dual trunk central retinal vein. Incidence in clinical practice. Arch Ophthalmol 1984;102:85-7.

9 Mann I. The development of the human eye. 3rd ed. London: British Medical Association, 1969:228.

10 Jackson E. Cilio-retinal and other anomalous retinal vessels. Ophthalmic Rev 1911;30:264-9.

11 Brown GC, Shields JA. Cilioretinal arteries and retinal arterial occlusion. Arch Ophthalmol 1979;97:84-92.

12 Lee SS, Schwartz B. Role of the temporal cilioretinal artery in retaining central retinal visual field in open-angle glaucoma. Ophthalmology 1992;99:696-9.

13 Hayreh SS, Zimmerman B, Podhajsky P. Incidence of various types of retinal vein occlusion and their recurrence and demographic characteristics. Am f Ophthalmol 1994;117: $429-41$. 